\title{
Avaliação visual da qualidade da estrutura do solo em sistemas de uso das terras ${ }^{1}$
}

\author{
Joelcio Eurich ${ }^{2}$, Pedro Henrique Weirich Neto ${ }^{3}$, Carlos Hugo Rocha ${ }^{4}$, Zíngara Rocio dos Santos Eurich ${ }^{5}$ \\ http://dx.doi.org/10.1590/0034-737X201461060017
}

\begin{abstract}
RESUMO
Sistemas sustentáveis de produção agropecuária demandam solos com boa estrutura, por isso, são necessários métodos eficientes para avaliação dessa estrutura. Objetivou-se, com este estudo, aplicar a metodologia de avaliação visual da estrutura do solo, em áreas previamente cobertas por Floresta com Araucária, em longo período de uso após o desmatamento. Selecionaram-se áreas sob três tipos de usos: floresta nativa, pastagem e cultivo agrícola, considerados como tratamento, sendo realizadas dez amostragens por tipo de uso. Nas amostras coletadas, procedeu-se à avaliação da qualidade da estrutura do solo, seguida da análise estatística dos dados, por meio da análise de variância e do teste de separação de médias. Observaram-se diferenças significativas nos valores médios de escore entre as áreas avaliadas. Os solos da área com floresta apresentaram Qe (Qualidade visual) médio = 1.68, estatisticamente semelhante ao dos solos sob pastagem $($ Qe médio $=2.25$ ) e inferior ao dos solos cultivados, Qe médio $=2.40$. Os valores de Qe obtidos por avaliação visual da qualidade da estrutura do solo mostraram-se eficientes para identificar diferenças entre os sistemas de uso do solo.
\end{abstract}

Palavras-chave: escore visual do solo, agregação do solo, pastagem, plantio direto.

\section{ABSTRACT}

\section{Visual assessment of soil structure quality in land use systems}

Sustainable agricultural production systems require well-structured soils and, thus, efficient methods for the assessment of soil structure are required. The objective of this study was to apply the methodology of visual quality of the soil structure in areas previously covered by Araucaria Forest and long-term use after deforestation. The following areas under three types of land use were selected: forest, pasture and farming, considered as treatments, from which ten samples were collected by each type of land use. In the collected samples, the visual quality of soil structure was carried out followed by statistical analysis, by means of analysis of variance and mean separation test. Significant differences in mean scores were found among the assessed areas. The soils under forest cover showed a mean Ve (visual score $)=1.68$, statistically similar to the soil under pasture (mean $\mathrm{Ve}=2.25)$ and lower than the farmed soils, (mean $\mathrm{Ve}=2.40$ ). Values of visual score obtained by visual assessment of the quality of soil structure were effective to identify differences among land use systems.

Key words:aggregationof soil, grassland, no-tillage, visualscore of soil.

\footnotetext{
Recebido para publicação em 26/03/2013 e aprovado em 11/02/2014.

${ }^{1}$ Parte integrante do trabalho de tese de doutorado do primeiro autor.

${ }^{2}$ Engenheiro-Agrônomo, Mestre. Doutorando em Agronomia - Departamento de Solos e Engenharia Agrí́cola, Universidade Estadual de Ponta Grossa, Avenida General Carlos Cavalcanti, 4748, 84030-900, Ponta Grossa, Paraná, Brasil. joe.eurich@gmail.com (Autor para correspondência).

${ }^{3}$ Engenheiro Agrícola, Doutor. Departamento de Solos e Engenharia Agrícola, Universidade Estadual de Ponta Grossa, Avenida General Carlos Cavalcanti, 4748, 84030-900, Ponta Grossa, Paraná, Brasil. lama1@uepg.br

${ }^{4}$ Engenheiro-Agrônomo, Doutor. Departamento de Solos e Engenharia Agrícola, Universidade Estadual de Ponta Grossa, Avenida General Carlos Cavalcanti, 4748, 84030-900, Ponta Grossa, Paraná, Brasil. chrocha@uepg.br

${ }^{5}$ Geógrafa. Mestre. Doutoranda em Geografia - Universidade Estadual de Ponta Grossa, Avenida General Carlos Cavalcanti, 4748, 84030-900, Ponta Grossa, Paraná, Brasil.
} 


\section{INTRODUÇÃO}

Nas últimas décadas, as tecnologias voltadas ao campo apresentaram crescimento significativo, seja em soluções para problemas diagnosticados seja para diagnóstico de prováveis problemas. Inúmeras ferramentas, instrumentais ou analíticas, são apresentadas todos os dias, nas mais diferentes áreas do conhecimento agrícola (Eurich et al., 2010a).

Essas tecnologias acarretam diferentes manejos de sistemas agrícolas, alterando continuamente o meio agrícola e suas interações com os recursos naturais. Muitas vezes não são claras, porque não estão determinadas, as vantagens e desvantagens de se adotar essa ou aquela técnica. No que diz respeito à visão holística da sustentabilidade, faltam metodologias e informações sobre para que locais a determinação da influência exercida por essas alterações nos sistemas de manejo do solo seria fundamental no desenvolvimento de sistemas agrícolas sustentáveis (Dexter \& Youngs, 1992).

A perda da estruturação do solo limita o desenvolvimento das plantas; consequentemente, resulta redução no potencial produtivo (Richart et al., 2005). Segundo Dexter (2004), as transformações e a consequente má qualidade física-estrutural do solo pode ser observada, avaliando-se diversos fatores, como baixa aeração, baixa infiltração de água e sistema radicular reduzido, refletindo a degradação da estrutura do solo. Entre as ferramentas utilizadas para a quantificação da degradação da qualidade física do solo, a densidade e a porosidade são as mais comumente utilizadas (Silva et al., 2012).

Infelizmente, muitas dessas análises fundamentais para o entendimento, bem como para a busca por sistemas mais sustentáveis de produção, são de difícil execução ou onerosas. Assim, por causa da heterogeneidade do meio rural, observam-se problemas de alcance dessas ferramentas e, até mesmo, a não existência de ferramentas mais eficazes, em alguns setores rurais (Eurich et al., 2010a).

Em vista disso, ferramentas de análises de qualidade estrutural dos solos, que apresentem aplicabilidade e baixo custo necessitam ser aprimoradas. O método denominado Avaliação Visual da Estrutura do Solo - AVES (Ball et al., 2007; Guimarães et al., 2011) pode suprir essa necessidade, tanto em regiões temperadas quanto em regiões tropicais (Giarola et al., 2009).

Sendo assim, objetivou-se aplicar a metodologia de avaliação visual da estrutura do solo, em áreas previamente cobertas por Floresta com Araucária, em longo período de uso após o desmatamento.

\section{MATERIAL E MÉTODOS}

O experimento foi realizado na Chácara Alcerich, situada no município de Palmeira, PR, entre 25²3' 24,34"
S e $49^{\circ} 56^{\prime} 30,46^{\prime \prime} \mathrm{O}$, em altitude aproximada de $900 \mathrm{~m}$, inserida em uma colônia de agricultores familiares, que, de acordo com Eurich et al. (2010b), é formada, na sua maioria, por produtores que têm a pecuária leiteira como principal atividade. O município insere-se na região centro-sul do Paraná e o tipo climático regional, segundo a classificação de Köppen, é temperado úmido, com verão temperado $(\mathrm{Cfb})$, ou seja, com temperatura média, no mês mais frio, abaixo de $18{ }^{\circ} \mathrm{C}$ (mesotérmico), com verões frescos, com temperatura média no mês mais quente abaixo de $22{ }^{\circ} \mathrm{C}$ e sem estação seca definida (IAPAR, 2000).

A fitofisionomia predominante na região é composta de áreas de pastagens, perenes e anuais, bem como áreas com sucessão entre pastagens anuais e lavouras anuais, associados a fragmentos de Floresta Ombrófila Mista, em geral acompanhando cursos d'água. Existe ainda a presença de capões, que constituem pequenas áreas de vegetação arbustiva e arbórea, ilhadas nas áreas de campo, sendo que os capões mais desenvolvidos apresentam aspecto de mata da formação de Araucárias (Rocha et al., 1993).

A propriedade em estudo iniciou suas atividades na década de 80 , após a derrubada da mata de capões acima descrita, como demonstrado na Figura 1. Foram selecionadas três áreas, conforme sistema de uso antrópico: área de mata nativa, área de pastagem e área de lavoura (Figura 2). Seus respectivos históricos de produção são apresentados na Tabela 1.

A área de mata amostrada permanece inalterada, assim preservando as características naturais, em termos qualitativos de estruturação do solo. A área de pastagem é caracterizada pela presença de braquiária (Brachiaria humidicula Rendle Schweick), sendo realizada no início do inverno a sobressemeadura de aveia preta (Avena strigosa Schreb) e de azevém (Lolium multiflorum Lam), sem o revolvimento do solo. A área de lavoura é manejada como pastagem durante o inverno, sendo semeados a aveia preta e o azevém, com o auxílio da gradagem. Durante o período de verão, é cultivada com grãos (milho com finalidade de silagem, rotacionado com soja no ano de 2007), em sistema de plantio direto.

A área de lavoura é utilizada para pastoreio durante o período de inverno, com lotação média de $5 \mathrm{UA} \mathrm{ha}^{-1}$, permanecendo no pastoreio por um período médio de 10 dias, com intervalos, entre pastoreios, de 30 dias. A área de pastagem é utilizada o ano inteiro, com uma lotação média de $5 \mathrm{UA} \mathrm{ha}^{-1}$, permanecendo no pastoreio por um período médio de 10 a 15 dias, com intervalos, entre pastoreios, de 30 a 50 dias.

Para a realização da avaliação visual da estrutura do solo, utilizou-se a metodologia proposta por Ball et al. (2007) e descrita por Giarola et al. (2009) e Guimarães et 
al., (2011). Foram abertas, com pá reta, minitrincheiras com 0,30 m de largura x 0,40 m de comprimento x 0,30 $\mathrm{m}$ de profundidade, para a extração de amostras indeformadas (blocos), da camada superficial do solo de cada tratamento, com $0,15 \mathrm{~m}$ de largura $\mathrm{x} 0,25 \mathrm{~m}$ de profundidade $\mathrm{x} 0,10 \mathrm{~m}$ de espessura.
Nas amostras coletadas, procedeu-se à avaliação da estrutura do solo, apoiando-se na aparência, na resistência e nas características das unidades estruturais de blocos de solo, sendo realizados dez pontos de amostragem em cada área, totalizando assim 30 pontos de avaliação. Foram utilizados cinco qualidades visuais (Qe) para a classificação da qualidade: de Qe = 1 (melhor qualidade estru-
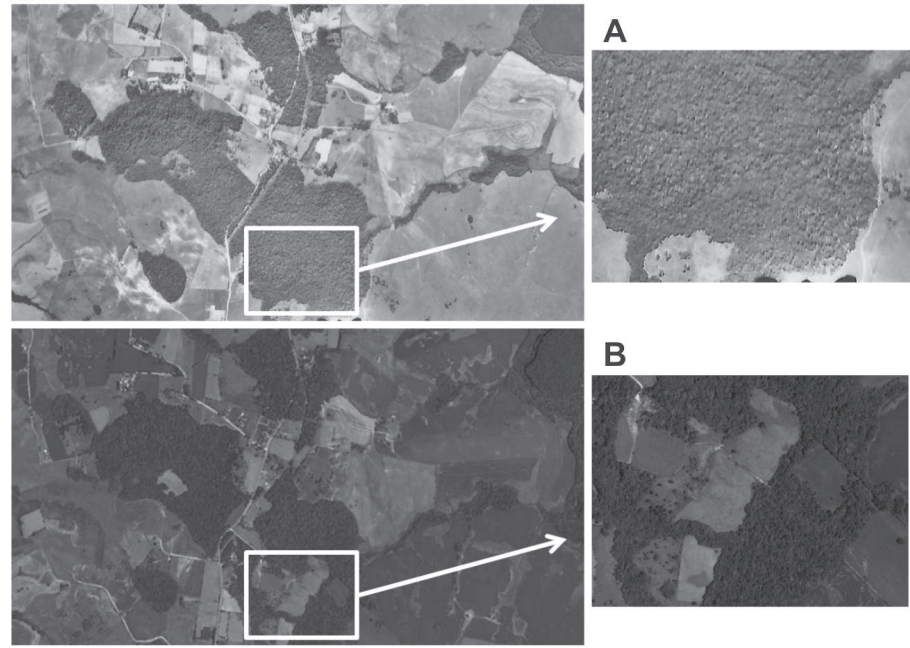

Figura 1. Área de estudo para a avaliação visual da qualidade da estrutura do solo. (a) antes do desmatamento - imagem de 1980 e (b) 30 anos pós-desmatamento - imagem de 2010 (Fonte: Google Earth ${ }^{\circledR}$ ).

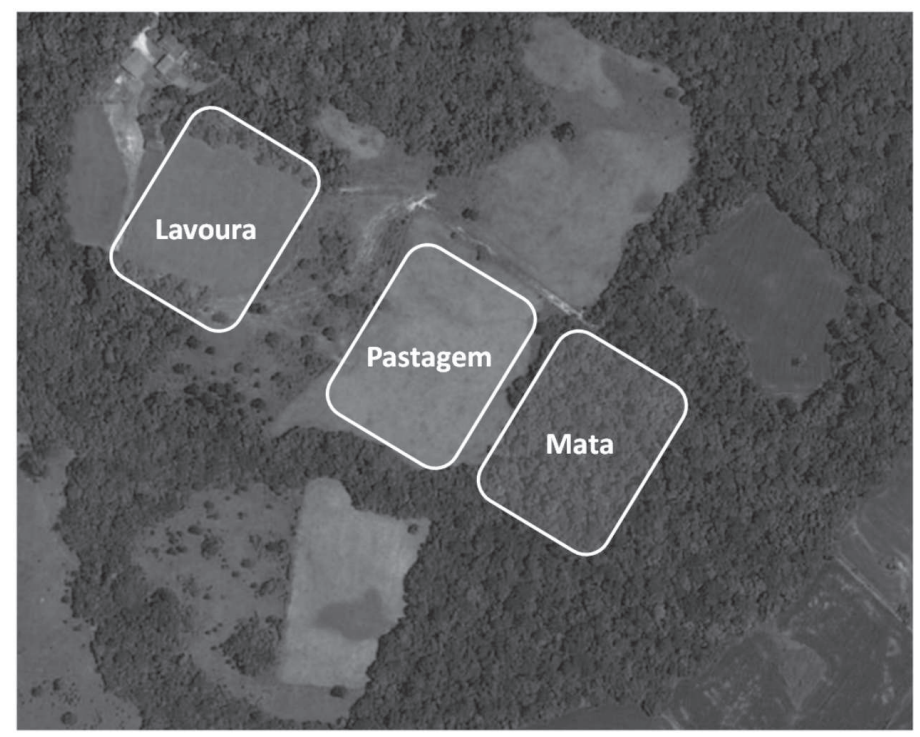

Figura 2. Áreas de estudo para a avaliação visual da qualidade da estrutura do solo.

Tabela 1. Áreas analisadas e suas respectivas culturas durante os últimos cinco anos

\begin{tabular}{|c|c|c|c|c|c|c|c|c|c|c|}
\hline \multirow{2}{*}{ Área } & \multicolumn{2}{|c|}{2007} & \multicolumn{2}{|c|}{2008} & \multicolumn{2}{|c|}{2009} & \multicolumn{2}{|c|}{2010} & \multicolumn{2}{|c|}{2011} \\
\hline & I & $\mathbf{V}$ & I & $\mathbf{V}$ & I & $\mathbf{V}$ & I & $\mathbf{V}$ & I & $\mathbf{V}$ \\
\hline Mata & \multicolumn{2}{|c|}{-} & \multicolumn{2}{|c|}{-} & \multicolumn{2}{|c|}{-} & \multicolumn{2}{|c|}{-} & \multicolumn{2}{|c|}{-} \\
\hline Pastagem & $\mathrm{Ss} \mathrm{Ap/Az}$ & Bp & $\mathrm{Ss} \mathrm{Ap} / \mathrm{Az}$ & $\mathrm{Bp}$ & Ss Ap/Az & $\mathrm{Bp}$ & Ss Ap/Az & $\mathrm{Bp}$ & $\mathrm{Ss} \mathrm{Ap} / \mathrm{Az}$ & $\mathrm{Bp}$ \\
\hline Lavoura & $\mathrm{S} A p / A z$ & So & S Ap/Az & Ms & $\mathrm{S} A p / A z$ & Ms & S Ap/Az & Ms & S Ap/Az & $\mathrm{Ms}$ \\
\hline
\end{tabular}

I-Inverno; V - Verão; Ss - Sobressemeadura; S - Semeadura; Ap - Aveia preta; Az - Azevém; Bp - Braquiária perene; So - Soja; Ms - Milho silagem. 
tural) a Qe $=5$ (pior qualidade estrutural) (Ball et al., 2007; Guimarães et al., 2011).

De posse dos dados, procedeu-se à análise estatística com o software estatístico SASM-Agri® (Canteri et $a l ., 2001)$, sendo realizada a análise de variância e posterior separação de médias pelo teste de Tukey, a 5\% de probabilidade.

\section{RESULTADOS E DISCUSSÃO}

A diferença de Ev entre os tratamentos (áreas) avaliados mostrou-se significativa a 5\% de probabilidade (Tabela 2). Observou-se um CV (Coeficiente de Variação) de 26,10\%, o que, de acordo com Pimentel-Gomes (1992), pode ser classificado como alto. Grande contribuição para altos valores de CV advém da área de pastagem estudada, que apresentou valores da qualidade visual (Qe) entre 1,52 e 3,65 (dados não apresentados), possivelmente influenciados pelo pisoteio animal desuniforme no momento do pastoreio.

Na Figura 3, observam-se as diferenças visuais entre as áreas estudadas, sendo possível identificar as diferentes formas de estruturação avaliadas pela metodologia. Na área de ambiente natural (mata - a), observou-se uma aparente deposição de serrapilheira, como substrato de matéria orgânica e sua contribuição na estruturação superficial do solo, sendo o elemento carbono o principal agente na melhoria da qualidade estrutural desses solos (Hickmann \& Costa, 2012). Na área de pastagem (b), observou-se uma uniformidade de estruturação ao longo do bloco, o que lhe conferiu

Tabela 2. Análise de variância simples dos escores de avaliação visual da qualidade da estrutura do solo

\begin{tabular}{lccccccc}
\hline Causa da variação & G.L. & S.Q. & Q.M. & F & F (5\%) & F (1\%) & significativo (5\%) \\
\hline Tratamentos & 2 & 2,88 & 1,44 & 4,73 & 3,35 & 5,48 & \\
Resíduo & 27 & 8,21 & 0,30 & & & & \\
Total & 29 & 11,09 & & & & & \\
\hline C.V. \% & 26,1 & & & & & & \\
\hline
\end{tabular}

C.V. - coeficiente de variação, G.L. - grau de liberdade, S.Q -soma dos quadrados, Q.M. - Quadrado médio
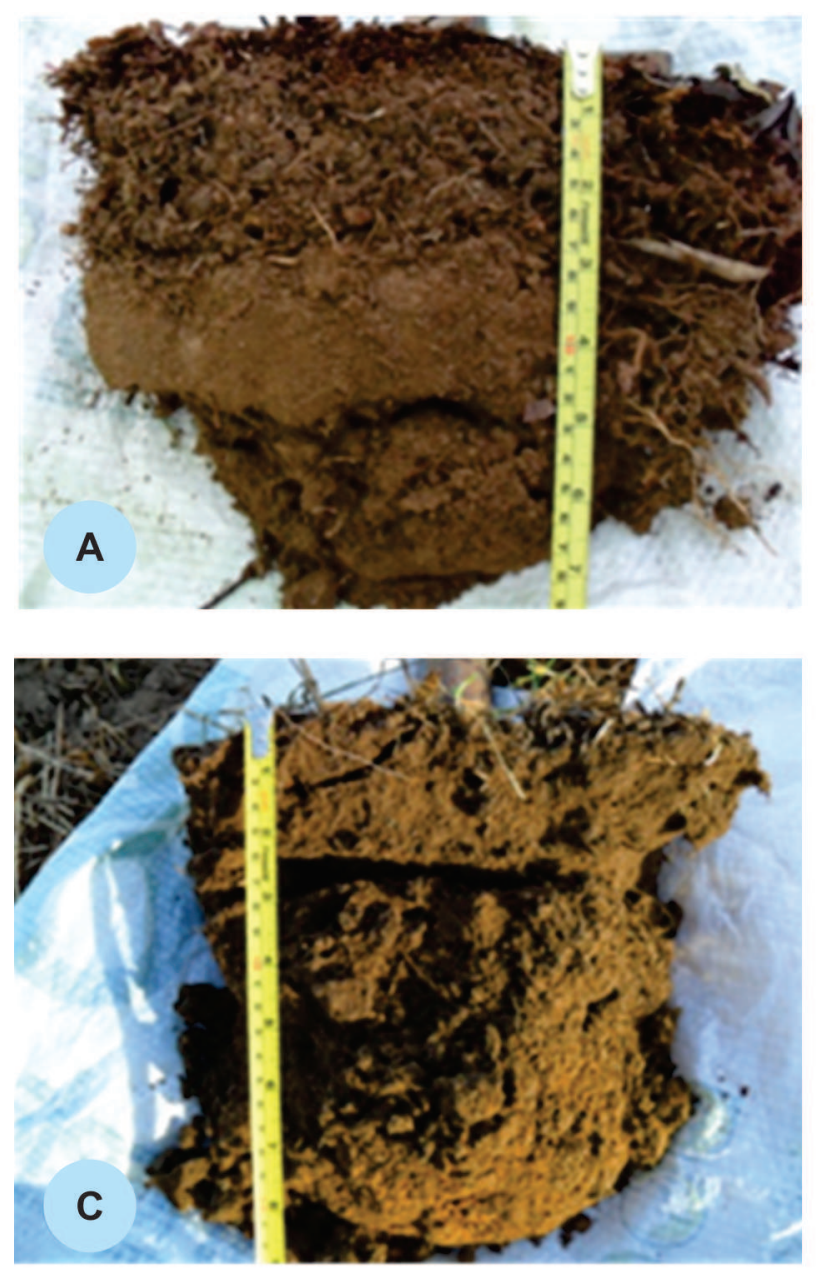

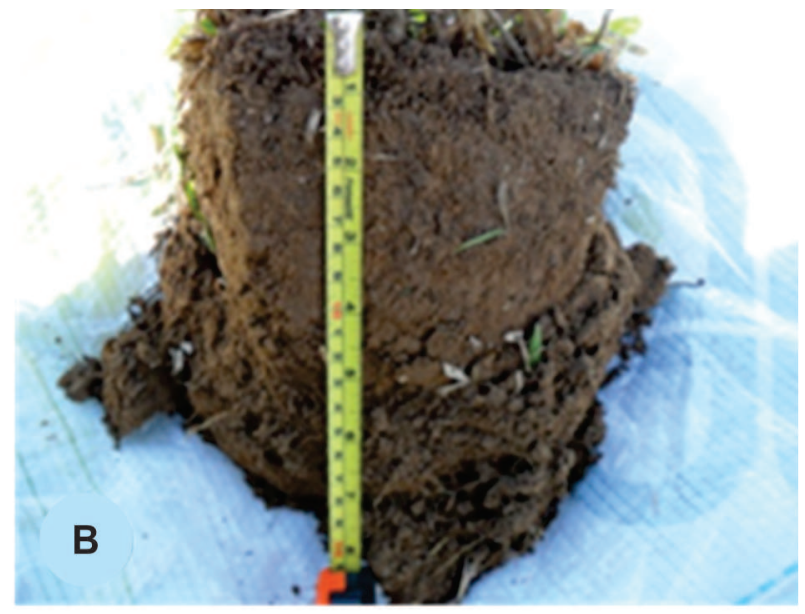

Figura 3. Blocos de solo extraídos para a avaliação visual da qualidade da estrutura do solo. (a) área de mata, (b) área de pastagem e (c) área de lavoura. 


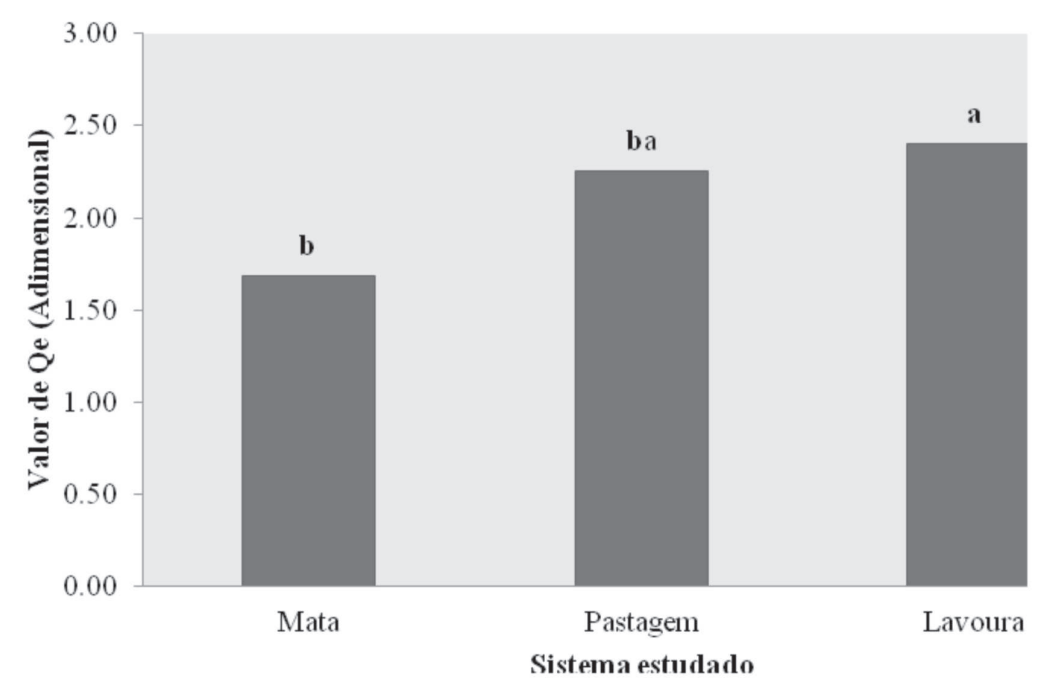

Figura 4. Qualidade visual (Qe) obtido nas áreas de mata, pastagem e lavoura. Barras seguidas da mesma letra não diferem entre si pelo Teste de Tukey a 5\% de probabilidade.

Qe médio. Na área de lavoura (c), identificou-se, em quase todas as amostragens, uma camada superior (cerca de $6 \mathrm{~cm}$ ) bastante compactada. Também foi observada no limite da extração do bloco de solo, sendo em média de $20 \mathrm{~cm}$ de profundidade, uma camada bastante adensada, que dificultava a introdução da pá para a coleta dos blocos. Essa limitação foi devida, possivelmente, ao manejo de máquinas realizado na área, tanto para a semeadura de aveia preta e azevém, na pastagem, durante o inverno quanto para realização da ensilagem de milho, causando assim uma compactação subsuperficial (Rosa et al., 2011).

A intensificação do uso da terra, com a transformação de ambientes naturais em ambientes manipulados anualmente para a atividade agropecuária, tende a transformála em ambientes pouco favoráveis a essas atividades ao longo do tempo, se não forem tomados cuidados para a manutenção das qualidades física, química e biológica dos solos (Caten et al. 2012).

Observaram-se diferenças significativas nos valores médios de escore entre as áreas avaliadas (Figura 4). A área com ambiente de mata apresentou uma Qe média de 1,68 , semelhante estatisticamente à da área de pastagem (Qe médio de 2,25) e inferior à da área de lavoura que apresentou uma Qe médio de 2,40.

Os valores de Qe obtidos na área de mata assemelham-se aos obtidos por Giarola et al. (2009), que encontraram valor médio de 1,70, e Giarola et al. (2010) com valor médio de 1,80. Esses autores também comentam a baixa variabilidade observada nos pontos de amostragem desse ambiente, fato observado neste trabalho, mostrando a boa estruturação uniforme em ambientes naturais. De acordo com Ball et al. (2007), valores de Qe entre 1 e 2 são considerados como de boa estruturação, faixa em que se encontra classificada o Qe da mata.
Os valores de Qe estatisticamente iguais entre as áreas de mata e de pastagem (Figura 4) mostram que o não revolvimento do solo acarreta o favorecimento de sua estruturação. O manejo de pastejo utilizado na área também favorece a boa condição de estruturação do solo, enfatizado pelos períodos de intervalos entre pastejos.

O sistema de lavoura, com um valor de Qe superior ao da área de mata (Figura 4), mostra que o atual sistema de manejo da área não favorece sua estruturação. O valor médio de Qe, observado, foi 2,40 , numericamente bem inferior ao encontrado por Giarola et al. (2009), em área de integração lavoura-pecuária ( $Q$ e $=3,07)$, mostrando que a área estudada ainda se apresenta com boa estruturação, em relação à de outros sistemas de manejo.

Müller et al., (2012), estudando áreas de longo período de plantio direto na região de Guarapuava - PR, observaram valores médios de Qe da ordem de 2,04, nas camadas superficiais $(0-15 \mathrm{~cm})$, mostrando que, em sistemas de lavoura sob plantio direto bem conduzidos, a estruturação do solo pode ser favorecida. Valores ainda melhores foram obtidos por Müller et al., (2009), que observaram médias de $\mathrm{Qe}=1,71$ para áreas sem revolvimento do solo, em experimentos de longa duração (mais de 10 anos), na China, Canadá e Alemanha.

\section{CONCLUSÕES}

O manejo de lavoura foi o que apresentou uma estruturação inferior à do ambiente de mata, sendo que o ambiente de pastagem sem revolvimento apresentou estruturação semelhante à do ambiente natural, embora todos os valores de Qe possam ser considerados bons. 
Os valores de escores visuais obtidos por avaliação visual da estrutura do solo foram eficientes para mostrar a diferença entre os sistemas de manejo.

\section{REFERÊNCIAS}

Ball BC, Batey T \& Munkholm LJ (2007) Field assessment of soil structural quality - a development of the Peerlkamp test. Soil Use and Management, 23:329-337.

Canteri MG, Althaus RA, Virgens Filho JS, Giglioti EA \& Godoy CV (2001) SASM - Agri : Sistema para análise e separação de médias em experimentos agrícolas pelos métodos Scoft Knott, Tukey e Duncan. Revista Brasileira de Agrocomputação, $1: 18-24$.

Caten AT, Minella JPG \& Madruga PRA (2012) Desintensificação do uso da terra e sua relação com a erosão do solo. Revista Brasileira de Engenharia Agrícola e Ambiental, 16:1006-1014.

Dexter AR (2004) Soil physical quality. Part I. Theory, effects of soil texture, density, and organic matter, and effects on root growth. Geoderma, 120:201-214.

Dexter AR \& Youngs IM (1992) Soil physic toward 2000. Soil and Tillage Research, 24:101-106.

Eurich J, Weirich Neto PH, Rocha CH, Ribeiro DRS \& Leitão K (2010a) Etnoagronomia: Saberes Vernaculares para determinação de zonas de manejo. In: Congresso Brasileiro de Agricultura de Precisão (ConBAP), Ribeirão Preto. CD-ROM.

Eurich J, Spies A, Weirich Neto PH \& Rocha CH (2010b) Tipificação de Agricultores Familiares produtores de Leite em uma colônia de Palmeira PR. In: I Congresso Sul Brasileiro de Produção Animal Sustentável (I ANISUS), Chapecó. CD-ROM.

Giarola NFB, Tormena CA, Silva AP \& Ball B (2009) Método de avaliação visual da qualidade da estrutura aplicado a Latossolo Vermelho Distroférrico sob diferentes sistemas de uso e manejo. Ciência Rural, 39:2531-2534.

Giarola NFB, Silva AP, Tormena CA, Ball B \& Rosa JA (2010) Visual soil structure quality assessment on Oxisols under notillage system. Scientia Agricola, 67:479-482.

Guimarães ML, Ball BC \& Tormena CA (2011) Improvements in the visual evaluation of soil structure. Soil Use and Management, 27:395-403.

Hickmann C \& Costa LM (2012) Estoque de carbono no solo e agregados em Argissolo sob diferentes manejos de longa duração. Revista Brasileira de Engenharia Agrícola e Ambiental, 16:1055-1061.

IAPAR - Instituto Agronômico do Paraná (2000) Cartas climáticas do Paraná: edição ano 2000. Versão 1.0. Londrina, IAPAR. CD-ROM.

Müller L, Kay BD, Deen B, Hu C, Zhang Y, Wolff M, Eulenstein F \& Schindler U (2009) Visual assessment of soil structure: Part II. Implications of tillage, rotation and traffic on sites in Canada, China and Germany. Soil \& Tillage Research, 103:188-196.

Müller MML, Tormena CA, Genú AM, Kramer LFM, Michalovicz L \& Caires EF (2012) Structural Quality of a No-Tillage Red Latosol 50 Months After Gypsum Aplication. Revista Brasileira de Ciência do Solo, 36:1005-1013.

Pimentel-Gomes F (1992) Curso de Estatística Experimental. 11 edição. Livraria Nobel S/A Editora. Piracicaba, ESALQ/USP. $121 \mathrm{p}$.

Richart A, Tavares Filho J, Brito OR, Llanillo RF \& Ferreira R (2005) Compactação do solo: Causas e efeitos. Semina, 26:321344
Rocha HO, Carrilho PF, Rocha CH \& Manni MR (1993) Aplicação da metodologia soter para levantamento semi-detalhado de solo (1:50.000) na região de Quero-Quero do município de Palmeira-PR. In: $7^{\circ}$ Simpósio Brasileiro de Sensoriamento Remoto (SBSR) Curitiba. Anais. CD-ROM

Rosa DP, Reichert JM, Mentges MI, Barros CAP, Reinert DJ \& Vieira DA (2011) Cultivo mínimo: Efeito da compactação e deformação abaixo da atuação da ponteira do subsolador. Revista Brasileira de Engenharia Agrícola e Ambiental, 15:1199-1205.

Silva BM, Oliveira GC, Serafim ME, Silva EA \& Oliveira LM (2012) Índice $\mathrm{S}$ no Diagnóstico da Qualidade Estrutural de Latossolo Muito Argiloso Sob Manejo Intensivo. Bioscience Journal, 28:338-345. 